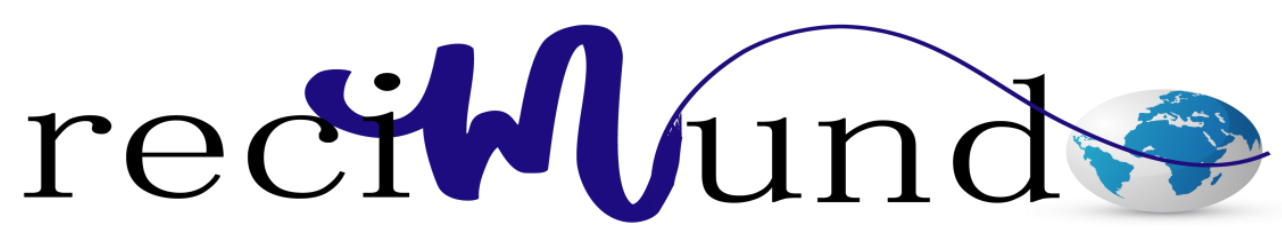

Revista Cientifica Mundo de la Investigación y el Conocimiento

Ing. Steffany Katherine Montecé Ochoa ${ }^{\text {a }}$, CPFG-EM. Francisco Javier Vásquez Torres ${ }^{\text {b}}$; TNNV-SS. Walter Javier Aguirre Bazurto ${ }^{\text {c }}$; TNFG-GC. Geovanny Gabriel Aguirre Bazurto ${ }^{\mathrm{d}}$

Estudio exploratorio del perfil del Microempresario en la ciudad de Guayaquil: Revisión de Literatura

Revista Científica Mundo de la Investigación y el Conocimiento. Vol. 1 núm., 5, diciembre, 2017, pp. 3-19

DOI: $10.26820 /$ recimundo/1.5.2017.3-19
a. steffanyka21@gmail.com
b. Armada del Ecuador; fvasquezt@armada.mil.ec
c. Armada del Ecuador; waguirreb@armada.mil.ec
d. Armada del Ecuador; gaguirre@armada.mil.ec 


\section{Estudio exploratorio del perfil del Microempresario en la ciudad de Guayaquil: Revisión de Literatura}

Vol. 1, núm. 5., (2017)

Ing. Steffany Katherine Montecé Ochoa; CPFG-EM. Francisco Javier Vásquez Torres; TNNVSS. Walter Javier Aguirre Bazurto; TNFG-GC. Geovanny Gabriel Aguirre Bazurto

\section{RESUMEN}

El presente trabajo de investigación se enfoca en conocer el perfil del microempresario en la ciudad de Guayaquil. Esta investigación tiene como objetivo determinar las principales características del microempresario para generar un perfil que aporte a futuras investigaciones y que contribuya al conocimiento de la sociedad. El mismo tiene un enfoque cualitativo y utiliza como método de recolección de información la entrevista a profundidad semiestructurada. Para el análisis de los datos se utilizó el sistema de codificación, que permitió simplificar los datos a través del estudio de la información entregada por los entrevistados, usando el software ATLAS TI para la verificación de los resultados obteniendo así resultados más exactos que permitió establecer el perfil del microempresario.

Palabras clave: Empresa; empresario; emprendimiento; perfil e innovación. 


\title{
Estudio exploratorio del perfil del Microempresario en la ciudad de Guayaquil: Revisión de Literatura
}

Vol. 1, núm. 5., (2017)

Ing. Steffany Katherine Montecé Ochoa; CPFG-EM. Francisco Javier Vásquez Torres; TNNV-

SS. Walter Javier Aguirre Bazurto; TNFG-GC. Geovanny Gabriel Aguirre Bazurto

\begin{abstract}
The present research work focuses on knowing the profile of the microentrepreneur in the city of Guayaquil. The objective of this research is to determine the main characteristics of the microentrepreneur in order to generate a profile that contributes to future research and contributes to the knowledge of society. It has a qualitative approach and uses the semistructured depth interview as a method of gathering information. For the analysis of the data, the coding system was used, which simplified the data through the study of the information provided by the interviewees, using the ATLAS TI software to verify the results, obtaining more accurate results that allowed establishing the profile of the microentrepreneur.
\end{abstract}

Keywords: Company; entrepreneur; entrepreneurship; profile and innovation. 


\section{Estudio exploratorio del perfil del Microempresario en la ciudad de Guayaquil: Revisión de Literatura}

Vol. 1, núm. 5., (2017)

Ing. Steffany Katherine Montecé Ochoa; CPFG-EM. Francisco Javier Vásquez Torres; TNNVSS. Walter Javier Aguirre Bazurto; TNFG-GC. Geovanny Gabriel Aguirre Bazurto

\section{Introducción.}

La presente investigación se puede definir como el análisis del comportamiento de un grupo de individuos de la ciudad de Guayaquil respecto a la administración de sus propios negocios con el objeto de determinar el perfil de estos como microempresarios, esto es considerando que las microempresas actualmente constituyen un segmento muy importante dentro de la economía ecuatoriana en términos de generación de ingresos y desarrollo de las regiones.

(Claudio, 2005) Sostiene que el 97\% del cierre de las microempresas es debido a una mala gestión de los administradores y esto generalmente es una de las principales causas o el error más común que cometen los microempresarios al momento de desarrollar sus actividades, es por ello que se puede decir que en la presente investigación se analizarán e interpretarán las principales razones del por qué al cierre forzoso de los negocios.

El presente trabajo de investigación abarca temas que nos ayudarán a comprender el problema más ampliamente e identificar cuáles son los principales factores que afectan a los microempresarios, detectar las causas del problema y entender la importancia de crear un perfil del mismo. Expondremos sobre las teorías que existen así como también la explicación de los conceptos de: empresa, empresario, emprendimiento, perfil e innovación, siendo imprescindible destacar que este estudio se sustenta en trabajos de tesis de pregrado y postgrado de diferentes universidades de Latinoamérica y del Ecuador, de las que se ha recolectado información relevante, que aportan significativamente a este estudio. 


\section{Estudio exploratorio del perfil del Microempresario en la ciudad de Guayaquil: Revisión de Literatura}

Vol. 1, núm. 5., (2017)

Ing. Steffany Katherine Montecé Ochoa; CPFG-EM. Francisco Javier Vásquez Torres; TNNVSS. Walter Javier Aguirre Bazurto; TNFG-GC. Geovanny Gabriel Aguirre Bazurto

Se desarrolla la metodología utilizada, destacando que la misma tiene un enfoque de tipo cualitativo, porque se considera es la mejor manera de buscar y conocer las características del comportamiento de un particular respecto a la administración de un negocio propio, a los fines de definir así su perfil. Las técnicas de investigación que utilizamos se formularán por el método de observación semiestructurada y participante, es decir, entrevistas a profundidad. Para la recolección de datos se les preguntó lo esencial, mientras se les observó y escuchó con detenimiento, siempre por respeto al valioso tiempo que se nos brindó, tomando nota de lo que exactamente el entrevistado manifestó. El análisis de datos consistió en la recolección de la información proporcionada por los entrevistados, desensamblar los mismo con etiquetas a un nivel conceptual, reensamblarlos proponiendo arreglos jerárquicos, interpretar los datos de manera completa y precisa para no crear desconcierto y como fase final la de generar las conclusiones respectivas. Para el análisis cualitativo, se utilizó la técnica que se basa en agrupar por categorías todas las ideas y temáticas principales expresadas por los entrevistados, comprendida en la presente investigación, por la expresión de 30 microempresarios. Se manipuló el análisis de codificación para la simplificación de la información del perfil de los microempresarios y además fue analizada por un software ATLAS TI para reagrupar y organizar datos cualitativos. Finalmente, todos los resultados se mostrarán en un documento que resuma el perfil exploratorio del microempresario y desarrollaremos la propuesta de perfil del microempresario en la ciudad de Guayaquil. 


\section{Estudio exploratorio del perfil del Microempresario en la ciudad de Guayaquil: Revisión de Literatura}

Vol. 1, núm. 5., (2017)

Ing. Steffany Katherine Montecé Ochoa; CPFG-EM. Francisco Javier Vásquez Torres; TNNVSS. Walter Javier Aguirre Bazurto; TNFG-GC. Geovanny Gabriel Aguirre Bazurto

\section{Materiales y Métodos.}

El tipo de estudio y alcance son concomitantes del diseño de la investigación. Para este caso en particular, se manifiesta que es una investigación cualitativa; según el alcance es: descriptiva, exploratoria. Debido al objetivo de estudio, se puede definir que la investigación será cualitativa, y; según el tipo de estudio, descriptiva.

(Denzin \& Lincoln, 2012) Consideraron que la investigación cualitativa es un multimétodo y se define en enfoque interpretativo y naturalista dado que estudia cosas en su entorno natural y pretende interpretarlas y llevarlas a un entendimiento común. Siguiendo el mismo contexto se añade el tipo descriptiva porque una vez obtenida la información se procederá a ordenar las características de la realidad observada, con la finalidad de proporcionar los resultados del objeto de estudio.

El presente estudio es de tipo cualitativo porque busca conocer las características del microempresario y generar un perfil del mismo, por esto el primer enfoque consistió en buscar, recopilar y analizar información obtenida en la literatura de diferentes fuentes, como investigaciones reveladas en artículos científicos, tesis de pre grado y postgrado, libros electrónicos y físicos. Las técnicas de investigación se formularán por el método de observación semiestructurada y participante, entrevistas a profundidad. Basado en los preceptos anteriores se discurre que el trabajo presente es de aplicación cualitativa ya que estudia la fenomenología en modo natural. 


\section{Estudio exploratorio del perfil del Microempresario en la ciudad de Guayaquil: Revisión de Literatura}

Vol. 1, núm. 5., (2017)

Ing. Steffany Katherine Montecé Ochoa; CPFG-EM. Francisco Javier Vásquez Torres; TNNV-

SS. Walter Javier Aguirre Bazurto; TNFG-GC. Geovanny Gabriel Aguirre Bazurto

Así mismo, ésta investigación es de tipo descriptiva, ya que siguiendo a (Bayardo, 1987)

se puede decir que la investigación descriptiva busca obtener información medible del estado actual del fenómeno de estudio, por lo tanto, busca detallar la correlación de sus variables. También es exploratoria, debido a que en este sentido, (Namakforoosh, 2005) asegura que las investigaciones exploratorias se sitúan en una perspectiva general del problema.

Este estudio tiene un alcance descriptivo y exploratorio, es decir, (Sampieri, Fernández, \& Baptista Lucio, 2006) mencionó que el alcance de una investigación se mide la profundidad del conocimiento que se desea lograr a través de la recolección de datos, con el fin de establecer pautas de comportamientos o establecer teorías.

(Maya, 2014) refiere que el método consiste en un proceso racional e inteligente de obtener respuesta a una serie de dudas o hipótesis, entendiendo su esencia y su relación con otros elementos, por lo tanto, el presente trabajo se realizará bajos los métodos: lógico, analítico y de síntesis, éstos dirigirán la ejecución y permitirá conocer de manera científica los antecedentes del microempresario de la ciudad de Guayaquil.

El método lógico se basa en la deducción, análisis y síntesis de la información, en cambio el método sistémico se dirige a moldear el objeto de estudio mediante la contextualización de sus componentes, así como la dinámica que existe entre ellos. Además, permite la selección de datos activos, que consiste en la descomposición representativa del objeto estudiado, lo cual se realizará mediante la entrevista en profundidad semiestructurada, la transcripción de información y el análisis para generar características que permitan identificar el perfil. 


\section{Estudio exploratorio del perfil del Microempresario en la ciudad de Guayaquil: Revisión de Literatura}

Vol. 1, núm. 5., (2017)

Ing. Steffany Katherine Montecé Ochoa; CPFG-EM. Francisco Javier Vásquez Torres; TNNVSS. Walter Javier Aguirre Bazurto; TNFG-GC. Geovanny Gabriel Aguirre Bazurto

Como punto de referencia (Hurtado \& Toro, 2007) mencionaron que "este tipo de razonamiento permite identificar todos los actores, factores y supuestos que inciden en el perfil micro empresarial"'.

\section{Resultados.}

\section{Empresa}

El término empresa ha sido ampliamente definido en la literatura y entre los principales autores mencionamos a (Chiavenato, 1992) quien definió a la empresa como un ente u organización social que está compuesto por la asociación de varias personas que trabajan por un fin común y que utilizan una variedad de recursos para poder convertir la materia prima en un producto terminado que está disponible a todas las personas de la sociedad logrando así obtener los beneficios esperados. Posteriormente, Cedeño (2005) planteó que el término empresa se refiere a un grupo, organización o entidad, que tienen un solo propósito y cuyas disposiciones se toman de manera libre y racionalmente y también comparó a las empresas con las personas, porque actúan como seres humanos, con libertad y convicción. Considerando las definiciones antes citadas el término empresa se refiere a la organización de personas que desempeñan distintos papeles, unos son propietarios, otros empleados y otros son clientes, la finalidad es atender requerimientos de las personas mediante productos o servicios y por esta acción generarse una retribución que generalmente se denomina ganancia. 


\section{Estudio exploratorio del perfil del Microempresario en la ciudad de Guayaquil: Revisión de Literatura}

Vol. 1, núm. 5., (2017)

Ing. Steffany Katherine Montecé Ochoa; CPFG-EM. Francisco Javier Vásquez Torres; TNNV-

SS. Walter Javier Aguirre Bazurto; TNFG-GC. Geovanny Gabriel Aguirre Bazurto

\section{Empresario}

Este término está relacionado con el emprendimiento que se refiere a iniciar una nueva actividad, también se vincula al término innovación por efectos del emprendimiento, para profundizar en la definición de empresario nos referiremos a Hisrich y Brush (1986) (Álvarez Martínez \& García del Junco, 1995) quienes definieron al empresario como un individuo que inicia su propio negocio y realiza una actividad comercial o de servicio a partir de una idea innovadora poniendo todo su esfuerzo y recursos en la explotación de un negocio con el objetivo de obtener recompensas monetarias. Andrade (2005) planteó al empresario como una persona natural o jurídica con ideas específicas para crear productos o servicios en los que asume el riesgo económico en caso de no ser aceptados por los clientes, esta es la principal característica que diferencia al empresario de un empleado. Posteriormente Ferrell, Hirt, Adriaenséns, Flores y Ramos (2010) establecieron el término empresario como una persona innovadora capaz de asumir riesgos tanto monetarios como materiales y que a pesar de sus adversidades y dificultades pone todo su esfuerzo en la creación de un producto o un servicio determinado para obtener beneficios.

Tomando como referencia los conceptos de empresario encontrados en la literatura, el término se puede resumir como, una persona que decide a partir de una idea iniciar una actividad de forma independiente para satisfacer alguna necesidad de las personas asumiendo el riesgo de pérdida económica, pero también espera lograr recompensa monetaria. 


\section{Estudio exploratorio del perfil del Microempresario en la ciudad de Guayaquil: Revisión de Literatura}

Vol. 1, núm. 5., (2017)

Ing. Steffany Katherine Montecé Ochoa; CPFG-EM. Francisco Javier Vásquez Torres; TNNVSS. Walter Javier Aguirre Bazurto; TNFG-GC. Geovanny Gabriel Aguirre Bazurto

\section{Emprendimiento}

El emprendimiento es un fenómeno cotidianamente observado en diferentes campos por ejemplo en la medicina, los negocios, la educación, entre las principales características se puede mencionar la de ejecutar por cuenta propia una idea que puede implicar materializarla con un producto o servicio o también no alcanzarla. También se puede referir el emprendimiento a tomar una nueva dirección, emprender un nuevo rumbo siempre con una idea preestablecida, para ampliar la definición nos referimos a las investigaciones de Schumpeter, (1934) definió el termino emprendimiento como una idea que es llevada a cabo por una persona o individuo que es el fundador de una nueva empresa en una idea innovadora que rompe con la forma tradicional de hacer las cosas. Posteriormente Ries, Kundel, (1991) definió al emprendimiento como la acción $\mathrm{u}$ actividad emprendedora que es desarrollada por una persona que es capaz de pensar en ideas y convertirlas en algo real aprovechando las oportunidades que se presenten frente a ellos y persiguiendo un determinado fin económico. Ries (2008) definió al término emprendimiento como la acción de emprender que a su vez proviene de una idea lógica y coherente que se hace tangible después de la mentalización de la misma.

En síntesis el término emprendimiento se refiere a desarrollar una idea innovadora generando nuevos productos, servicios o formas de ejecutar una actividad, generalmente se busca obtener un beneficio pero existe el riesgo de no conseguir los resultados deseados y perder los recursos económicos. Igualmente se puede definir como la actitud que toma el individuo para llevar a cabo una idea o proyecto mentalizado y estructurado con anterioridad y que a su vez es 


\section{Estudio exploratorio del perfil del Microempresario en la ciudad de Guayaquil: Revisión de Literatura}

Vol. 1, núm. 5., (2017)

Ing. Steffany Katherine Montecé Ochoa; CPFG-EM. Francisco Javier Vásquez Torres; TNNVSS. Walter Javier Aguirre Bazurto; TNFG-GC. Geovanny Gabriel Aguirre Bazurto

gestionado por una persona llamado emprendedor que realiza un esfuerzo haciéndole frente a las dificultades del mercado para alcanzar una meta u objetivo.

\section{Perfil}

Cuando se menciona la palabra perfil, es posible que las personas imaginen características individuales o relieves de algún lugar geográfico, es decir aspectos principales que permiten hacerse una idea de una persona o lugar y en la literatura se observan varias definiciones para este término. En este sentido Bernstein (1991) definió el perfil como una imagen parcial en la que sobresalen los rasgos de una figura y también mencionó que el término ha sido distinguido como un ensayo que a pesar de orientarse en un punto selectivo sobre la materia o situación no deja de enfocarse sobre las particularidades esenciales de la misma. Posteriormente (Alles, 2006) definió el perfil como la parte descriptiva de puestos de trabajo basado en recopilación de información de diferentes fuentes. Además, Davidson y Begley, (2012) detallaron el término perfil como un conjunto de rasgos peculiares que puede expresarse producto de las experiencias y para complementar la definición (Alles, 2006) mencionaron que el perfil se refiere a una serie de requisitos primordiales para el desempeño de un cargo específico y también debe ser definido de manera despersonalizada detallando todas las capacidades para ejecutarlo. Considerando las definiciones antes citadas podemos definir el perfil como los rasgos, características primordiales de algo o de alguien producto de la recolección de información necesaria suficiente que permitan su descripción. 


\section{Estudio exploratorio del perfil del Microempresario en la ciudad de Guayaquil: Revisión de Literatura}

Vol. 1, núm. 5., (2017)

Ing. Steffany Katherine Montecé Ochoa; CPFG-EM. Francisco Javier Vásquez Torres; TNNVSS. Walter Javier Aguirre Bazurto; TNFG-GC. Geovanny Gabriel Aguirre Bazurto

\section{Innovación}

La innovación se refiere a cambios que se introducen en el medio ambiente, puede ser en la vida cotidiana, en los negocios y en otros entornos, son ejemplos que manifiestan signos de innovación las nuevas ideas, propuestas, nuevos productos, servicios o procedimientos que se aplican en el mercado, para profundizar en la definición de innovación nos referiremos a diferentes autores como Podadera (1997) quien definió la palabra innovación como el proceso de generar nuevas ideas que después de un tiempo se desarrollarán como nuevos productos o procesos que serán incrustadas en la economía a través del mercado. Posteriormente, Jiménez (2003) definió el término innovación como un proceso previamente planificado y con fases definidas y además mencionó que en el entorno empresarial la innovación es una postura de posicionamiento más que un proceso estructurado y con una clara alineación al cambio. Años después, Romero (2012) definió a la innovación como el lanzamiento de un producto, bien o servicio, o de un proceso relativamente nuevo o mejorado y además puntualizó que se refiere a un método de comercialización nuevo a aplicarse a los negocios y finalmente, Gómez (2013) estableció la palabra innovación como una forma de encontrar y dar solución a los problemas existentes. En síntesis, la innovación es el proceso para generar nuevas ideas o mejorarlas que posteriormente se implementarán como productos o servicios en el mercado y es una forma de encontrar y dar solución a los problemas existentes.

Para estudiar el perfil del microempresario es relevante entender conceptos que se relacionan con el empresario, en este sentido abordamos los conceptos empresario, emprendimiento, innovación, empresa y perfil y llegamos a la conclusión de que estos conceptos 


\section{Estudio exploratorio del perfil del Microempresario en la ciudad de Guayaquil: Revisión de Literatura}

Vol. 1, núm. 5., (2017)

Ing. Steffany Katherine Montecé Ochoa; CPFG-EM. Francisco Javier Vásquez Torres; TNNVSS. Walter Javier Aguirre Bazurto; TNFG-GC. Geovanny Gabriel Aguirre Bazurto

constituyen un marco que permiten entender al empresario en sus diferentes manifestaciones. En resumen, la innovación es el proceso para generar nuevas ideas y posteriormente implementarlas. Otro concepto importante es el emprendimiento que en la literatura se lo define como la actitud del individuo para llevar a cabo una idea o proyecto mentalizado por una persona llamada emprendedor, que asume dificultades y riesgos con el objetivo de alcanzar una meta. El siguiente término al que nos vamos a referir es empresario que en la literatura se menciona identificando a una persona que decide a partir de una idea, iniciar una actividad de forma independiente para satisfacer alguna necesidad que conlleva riesgos de pérdidas económicas con el objetivo de obtener una recompensa monetaria. El siguiente paso para un empresario es organizarse y una de las formas más comunes de organización se denomina empresa que ha sido definida en la literatura como la coordinación de personas y recursos entorno a un objetivo regularmente se refiere a un beneficio económico, en este sentido aquí emergen términos como empleado, propietario, cliente y proveedor todos organizados alrededor del ente denominado empresa y finalmente nos referiremos al concepto del perfil que se refiere a los rasgos, características primordiales de algo o de alguien, producto de la recolección de información necesaria para su descripción.

\section{Conclusiones.}

Este trabajo de investigación cualitativa permitió extraer información sobre las características de los microempresarios para generar un perfil y se puede concluir que: 


\section{Estudio exploratorio del perfil del Microempresario en la ciudad de Guayaquil: Revisión de Literatura}

Vol. 1, núm. 5., (2017)

Ing. Steffany Katherine Montecé Ochoa; CPFG-EM. Francisco Javier Vásquez Torres; TNNVSS. Walter Javier Aguirre Bazurto; TNFG-GC. Geovanny Gabriel Aguirre Bazurto

Los microempresarios entrevistados de la ciudad de Guayaquil consideran que los empresarios son personas innovadoras, responsables y disciplinados y que los microempresarios se caracterizan como personas innovadoras, informales y con bajo nivel de capacitación.

Los microempresarios entrevistados no manejan los recursos tecnológicos hábilmente, por ende controlan información limitada e interactúan poco con las redes sociales. Esto se observa como una limitante para aprovechar esta vía de comunicación con los clientes, colaboradores y proveedores.

Los microempresarios entrevistados no realizan análisis de viabilidad al iniciar su negocio lo que indica que no miden si su proyecto es sostenible o rentable económicamente,

Los microempresarios entrevistados no invierten en publicidad necesaria para dar a conocer su negocio lo que proporciona una pérdida de posibles clientes potenciales.

\section{Recomendaciones.}

Este trabajo de investigación es un aporte para comprender al microempresario en la ciudad de Guayaquil a partir de sus características y comportamiento dentro del ambiente en donde se desarrolla, sin embargo, se recomienda:

Fomentar más investigaciones acerca de las características del microempresario para poder conocer un poco más sobre sus hábitos para administrar su negocio de esta forma entender sus necesidades lo cual podría aportar con información que permita desarrollar otros estudios con propuestas para solventar las necesidades del microempresario. 


\section{Estudio exploratorio del perfil del Microempresario en la ciudad de Guayaquil: Revisión de Literatura}

Vol. 1, núm. 5., (2017)

Ing. Steffany Katherine Montecé Ochoa; CPFG-EM. Francisco Javier Vásquez Torres; TNNV-

SS. Walter Javier Aguirre Bazurto; TNFG-GC. Geovanny Gabriel Aguirre Bazurto

Realizar estudios que permitan entender que impulsa a las personas a convertirse en microempresarios, esto podría aportar al entendimiento de este fenómeno.

Implementar desde el gobierno local, políticas, estrategias y estudios estadísticos más profundos que permitan ampliar y mejorar el entendimiento del perfil del microempresario. Lo que podría devenir en el desarrollo de propuestas comerciales de crédito y de apoyo gubernamental.

Llevar a cabo otros trabajos investigativos que comparen las principales características del microempresario con las del empresario para determinar sus diferencias y semejanzas que ayude a comprender el perfil de cada uno.

Efectuar estudios estadísticos que permitan determinar el número de microempresarios que sobreviven en tiempo promedio de duración de los negocios y cuantos microempresarios tienen periodos más cortos de duración en la ciudad de Guayaquil y en las diferentes regiones del Ecuador,

Investigar sobre el uso de las tecnologías de información y comunicación por parte de los microempresarios de la ciudad de Guayaquil y foráneos con la finalidad de entender la forma en que las aplican en sus actividades.

Desarrollar instrumentos de aprendizaje para que los microempresarios obtengan una mejor capacitación y alternativa en el futuro. 


\section{Estudio exploratorio del perfil del Microempresario en la ciudad de Guayaquil: Revisión de Literatura}

Vol. 1, núm. 5., (2017)

Ing. Steffany Katherine Montecé Ochoa; CPFG-EM. Francisco Javier Vásquez Torres; TNNVSS. Walter Javier Aguirre Bazurto; TNFG-GC. Geovanny Gabriel Aguirre Bazurto

Elaborar investigaciones que produzcan instrumentos de fácil comprensión y lectura en relación a los temas de interés de los microempresarios de manera que estos les pueda servir asesoramiento y consultoría antes del emprendimiento para disminuir futuros errores.

Estudiar las causas que inducen a las personas a convertirse en empresarios o microempresarios que proporcionen información o herramientas que permitan impulsar a cualquier persona a convertirse en emprendedores o microempresarios exitosos.

Investigar las diferencias por género y edad entre los microempresarios porque podrían presentarse diferencias en sus perfiles que pueden ser relevantes para el entendimiento y por ende ser útiles para el desarrollo de herramientas.

\section{Bibliografía}

Alles, M. (2006). Selección por competencias atracción y reclutamiento en las redes sociales: Entrevista y medición de competencias., (págs. 30-32).

Álvarez Martínez, P., \& García del Junco, J. (1995). Correlación entre los perfiles profesional y académico de los ejecutivos españoles. 44-51.

Bayardo, M. G. (1987). Introducción a la Metodologia de la Investigación Educativa II. Guadalajara, Jalisco, México: Progreso, S.A. de C.V.

Chiavenato, I. (. (1992). Clima Organizacional. Mdexico: Mc Graw Hill.

Claudio, S. (1 de NOVIEMBRE de 2005). Por qué fracasan las Pymes. Recuperado el 7 de AGOSTO de 2017, de https://www.gestiopolis.com/por-que-fracasan-las-pymes/

Denzin, N. K., \& Lincoln, Y. S. (2012). Manual de investigación cualitativa. (Vol. 1). Barcelona: Gedisa.

Hurtado, I., \& Toro, J. (2007). Paradigmas y métodos de investigación en tiempos de cambio. Valencia.

Maya, E. (2014). Métodos y Técnicas de Investigación. En J. Sosa - Martínez, Método Científico (págs. 11-88). 


\section{Estudio exploratorio del perfil del Microempresario en la ciudad de Guayaquil: Revisión de Literatura}

Vol. 1, núm. 5., (2017)

Ing. Steffany Katherine Montecé Ochoa; CPFG-EM. Francisco Javier Vásquez Torres; TNNV-

SS. Walter Javier Aguirre Bazurto; TNFG-GC. Geovanny Gabriel Aguirre Bazurto

Namakforoosh, M. N. (2005). Metodología de la investigación. Mexico: Limusa.

Sampieri, R. H., Fernández, C., \& Baptista Lucio, P. (2006). Metodología de la Investigación (Cuarta Edición ed.). Mexico: Mc Graw Hill Interamericana Editores S.A. de C.V. 